\title{
Nicotine Addiction: Neurobiology and Mechanism
}

\author{
Raj Kumar Tiwari', Vikas Sharma', Ravindra Kumar Pandey², Shiv Shankar Shukla* \\ ${ }^{1}$ Columbia Institute of Pharmacy, Raipur, C.G., India \\ ${ }^{2}$ Columbia Institute of Pharmacy, Raipur, C.G. India
}

\section{Key Words}

Nicotine, dopaminergic system, GABAergic, withdrawal, acetylcholine, MAO

\begin{abstract}
Nicotine, primary component of tobaco produces craving and withdrawal effect both in humans and animals. Nicotine shows a close resemblance to other addictive drugs in molecular, neuroanatomical and pharmacological, particularly the drugs which enhances the cognitive functions. Nicotine mainly shows its action through specific nicotinic acetylcholine receptors located in brain. It stimulates presynaptic acetylcholine receptors thereby enhancing Ach release and metabolism. Dopaminergic system is also stimulated by it, thus increasing the concentration of dopamine in nuclear accumbens. This property of nicotine according to various researchers is responsible for reinforcing behavioral change and dependence of nicotine. Various researchers have also depicted that some non dopaminergic systems are also involved for rewarding effect of nicotinic withdrawal.

Neurological systems such as GABAergic, serotonergic, noradrenergic, and brain stem cholinergic may also be involved to mediate the actions of nicotine. Further, the neurobiological pathway to nicotine dependence might perhaps be appropriate to the attachment of nicotine to nicotinic acetylcholine receptors, peruse by stimulation of dopaminergic system and activation of general pharmacological changes that might be responsible for nicotine addiction. It is also suggested that MAO A and $\mathrm{B}$ both are restrained by nicotine. This enzyme helps in
\end{abstract}

Received: May 09, 2019 Reviewed: May 29, 2019 Accepted: Dec 16, 2019

(C) This is an Open-Access article distributed under the terms of the Creative Commons Attribution Non-Commercial License (http://creativecommons.org/licenses/by-nc/4.0/) which permits unrestricted noncommercial use, distribution, and reproduction in any medium, provided the original work is properly cited. degradation dopamine, which is mainly responsible for nicotinic actions and dependence. Various questions remain uninsurable to nicotine mechanism and require more research. Also, various genetic methods united with modern instrumental analysis might result for more authentic information for nicotine addiction.

\author{
Abbreviations \\ GABA: Gamma-Aminobutyric acid, \\ MAO: Monoamineoxidase, \\ CNS: Central Nervous System, \\ CYP2A6: Cytochrome P2A6 enzyme, \\ CYP2B6: Cytochrome - P2B6 enzyme, \\ Ach: Acetylcholine, \\ nAChRs: Nicotinic acetylcholine receptors, \\ ACTH: Adrenocorticotropic hormone, \\ 5- HT: 5 hydroxyl trypatmine, \\ VTA: Ventral tegmental area, \\ NAc: Nucleus accumbens, \\ PFC: Prefrontal Cortex.
}

\section{Introduction}

Drug addiction is considered as the disorder of the brain reward system which is measured as multifarious disease of the CNS [1]. The preliminary events of addiction depict drug accomplishment on a specific place especially at neuronal junctions. The synapses are naturally activated and elicit the reinforcement activities of addiction. [2].

Nicotine is obtained from the leaves of tobacco ( $\mathrm{Ni}$ cotiana tabacum), is being used for centuries for its pleasurable effect. Nicotine was initially obtained from Nicotiana tabacum Posselt and Reimann in 1828. It was named after Jean Nicot, who first time introduced
"Corresponding Author

Shiv Shankar Shukla. Columbia Institute of Pharmacy, Raipur, C.G., India.

Tel: +91-982-612-4442

E-mail: shivpharma007@gmail.com 
Nicotiana tabacum to the French court in 1560 [3].

The mode of administration to nicotine may be through sniffing, chewing or by smoking. Nicotine comes in the category of alkaloid (1-methyl-2-[3-pyridyl] pyrrolidine) and is the major phytoconstituent responsible for addiction. Latest findings had revealed addiction created via nicotine is very influential and similar to addictions to abusive substances such as cocaine and heroin. [4].

In current times, the utilization of tobacco has made huge influence to younger generations and to a large part of humanity. If truth be told, Nicotiana is the entry substance to other substances of abuse like Heroine \& Ganja [5]. The noteworthy feature regarding Nicotiana application is with early administration it starts to show its action without any failure. As soon as youth attenuates administration of nicotine, is an indication and fear that youth might get trapped up in additional dangerous activities [10]. The tox- ic measure of nicotine is being predicted to be $40-60 \mathrm{mg}$, adult measure as $0.8-1 \mathrm{mg} / \mathrm{kg}$ and for children about $10 \mathrm{mg}$ (1 mg. kg) [6].

Majority $(70-80 \%)$ of Nicotiana is degraded to cotinine in individual by hepatosomal microenzymes, and cytochrome P450 2A6 (CYP2A6) is responsible for 90\% of this degradation [12]. Cotinine is additionally degraded by means of CYP2A6 to trans-3'-hydroxycotinine [7]. Nicotine is too metabolized to nor nicotine by means of $\mathrm{N}$-demethylation by CYP2A6 and CYP2B6 at various substance concentrations, correspondingly [8]. CYP2A6 and CYP2B6 assistance in the degradation of Nicotiana may rely on the appearance concentrations of these isoforms in liver of individual [9]. After chronic administration of it, plasma nicotine, cotinine and nornicotine quantity stay somewhat stable, whereas qunatity of nicotine and nornicotine in CNS rise about 4 times [10].

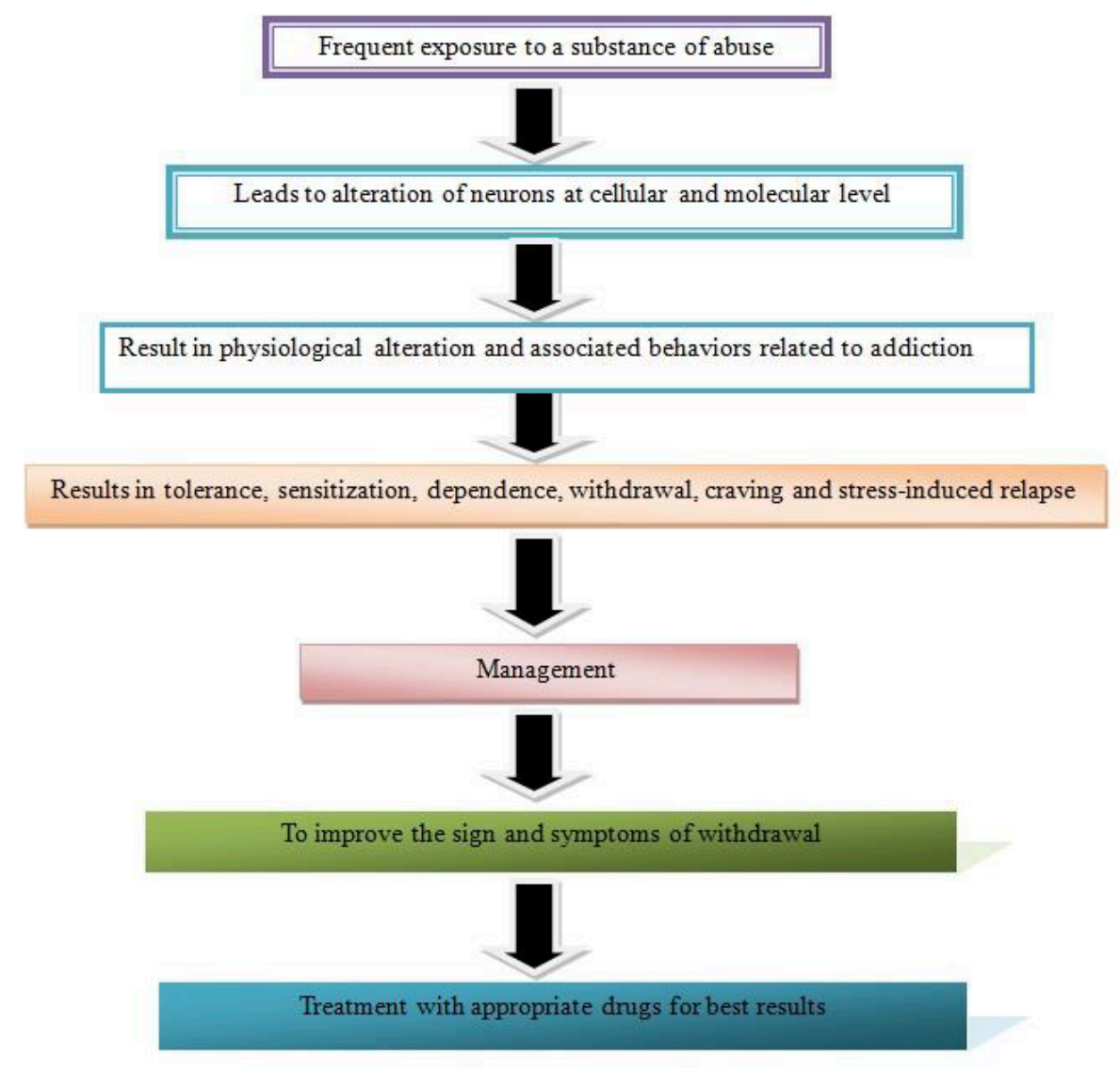

Figure 1 Physiology and management of Nicotine addiction 


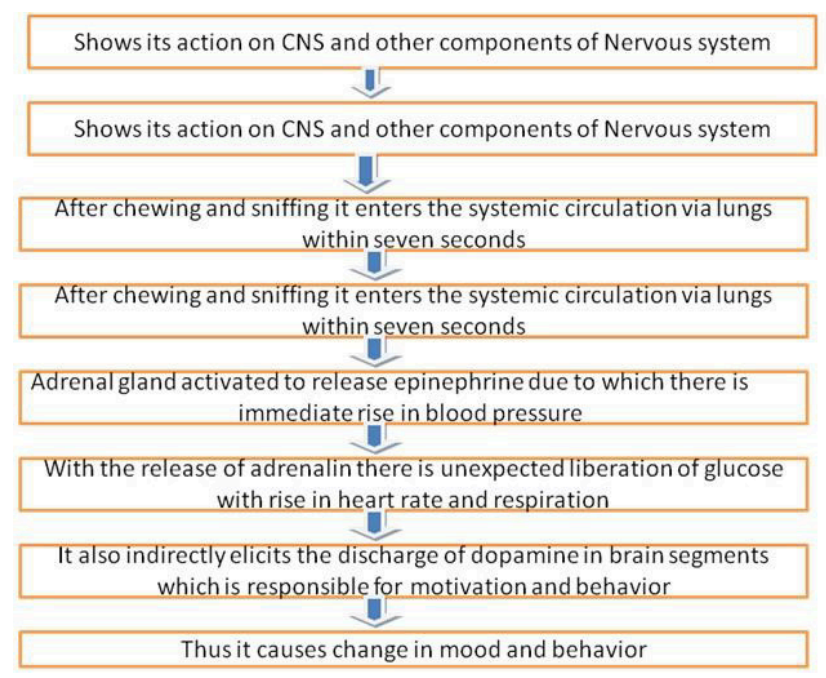

Figure 2 Physiology of Nicotine Action

\section{Pathways associated with nicotine addiction}

\subsection{Cholinergic system}

Newer investigation has revealed the action of nicotine on the CNS. Its action shows a majority of performance activity. It easily enters BBB stimulating nicotinic ACh receptors. These nicotinic cholinergic receptors act as a key ACh receptor subtypes [11]. Excitation of presynaptic nAChRs elicits the Ach discharge plus its metabolism. Long term exposures of nicotine produces receptor inactivation with successive up-regulation of nAChRs situate. Cholinergic receptors are located in several brain segments particularly located in the midbrain tegmentum, the striatum, nucleus accumbens and the ventral tegmentum. It is also present in muscles, adrenal glands, cardiovascular system and other body parts [12]. The activation of these receptors is usually done by the ACh mainly in CNS and neurons. These neurotransmitter and associated receptors are concerned with numerous actions, together with respiration, preservation of heart rate, reminiscence, attentiveness, and muscle retrenchment. In addition, requisite to AChRs, it too shows its presence in receptors of autonomic ganglia, adrenal medulla and the neuronal muscular connection [13]. The precise positions for attachment in CNS include hypothalamus, hippocampus, thalamus midbrain, brain stem and cerebral cortex. It moreover shows its presence in receptors of nigrostriatal and mesolimbic dopaminergic neurons. After stimulations of nicotine receptors it releases ACh, nor-epinephrine, dopamine, serotonin, vasopressin, growth hormone and ACTH [14]. Nicotiana is mainly potent activator of mid brain dopamine reward pathway. It also shows its action on locus ceruleus activating awareness, provocation, and attentiveness with anxiety activity producing the nicotine abusers extra attentive [15]. It moreover changes role of various neurotransmitters concerned with pathophysiology of various psychiatric chaos. It mainly comprises of serotonin (5-HT), glutamate, g-amino butyric acid (GABA), dopamine, endogenous opioid peptides and nor-epinephrine [16]. The outcomes created by it may be presynaptic, pre terminal or cell body nicotine receptors, moderately achieved during neurotransmission in which pre-synaptically discharged acetylcholine operate on postsynaptic, junctional nAChRs to create neuronal sacking [17].

\subsection{Dopaminergic System}

\subsubsection{The Ascending Mesolimbic and Meso- cortical Pathways}

Main ascending dopaminergic pathways instigate from cell bodies in the brainstem, mostly in the substantia nigra pars compacta and VTA.

\subsubsection{Nigrostriatal pathway}

The neurons from the substantia nigra elicits through the inner capsule largely to the striatum, thus creating the nigrostriatal Dopaminergic system, which reins motor activity and muscle tone [18].

\subsubsection{Mesolimbic pathway}

The mesolimbic dopaminergic system contains neurons which invent from the VTA and exceed through the medial forebrain bundle, protrudes the NAc, olfactory tubercle, and other limbic area such as the amygdale, hippocampus and septum. The mesolimbic system adds a key liability in the management of motivation, emotion, and motor activity. The system is too beneficial in mediating the satisfying properties of physically abused drugs. The NAc obtain the largest projections of the neurons from the VTA. Structurally the NAC contains of sub-territories, of this ventro medial shell and dorso- lateral core emerge to be leading. The shell, that obtains afferents from subcortical and brainstem compositions, exhibits better neuro-anatomical variety than the core. The shell receives outputs indication to the core via the feed-forward striatopallido-thalamocortico-striatal pathway. Both the shell and the core region emerge to be included in reward-related behavior [19].

\subsubsection{Mesocortical pathway}

The neuron which forms the mesocortical dopaminergic system comes out from the VTA, and they pass through to cortical regions together with the PFC, the entorhinal and cingulate cortices. This exacting brain system is concerned in management of elevated cognitive purposes. The PFC is also involved in drug-reward chronic ingestion of an abused drug and is recommended to create dopaminergic hypofunction in the PFC that ought to lie beneath impulsivity and failure of management of drugcarving behavior [20]. 


\section{Neuropharmacology of nicotine addiction}

\subsection{Action on dopaminergic transmission}

There is significant proof to recommend that nicotine participates in sustaining the tobacco smoking practice and a lot of regular smokers turn out to be reliant on nicotine. It is a dominant carving substance in human being. Mechanism by which it elicits compulsion stays uncertain [21]. Behavioral research in animals does specify that it is a compulsive substance that adds force to self-administration. The property of nicotine by the virtue of corroboration and behavioral uptaking is mainly done via mesolimbic dopamine system [22]. It constitutes of cell bodies of neurons restricted in the ventral tegmental area (VTA) and axons entering the NAc and PFC. It are therefore named as mesolimbic and mesocortical projections, correspondingly. The mesolimbic dopamine system gets activated by the presence of nicotinic receptors VTA and NAc[23].

This system is considered accountable for the reinforcing operations such as dependence and reliance activity of Nicotiana. So, nicotine shows somewhat similar actions to other addictive substances like cocaine, amphetamine, alcohol, and opiate. The Ventral tagmental area and associated neuron protuberances to NAc are supposed to be implicated in reward with the craving property of abusive substance [24].

Recent research findings showed that dopamine is the key chemical in the brain which is implicated in intervene the need to use abusive drug. In mind, it excites the discharge of dopamine into gratification track. The extracellular concentration of dopamine is enhanced in NAcs by nicotine. [25]. The disruption of dopamine neuron in mesolimbic segments has given us proof for uptake of nicotine by themselves in rats. It too assuages locomotory stimulant result of injected nicotine [26]. Administrations of nicotine or nicotine agonist's subcutaneously to VTA might effect in improved locomotion. The cell bodies of dopamine in VTA mianly show the effect of nicotine. Administration of agonist of nicotine subcutaneously into the dopaminergic segments of NAc attenuates dopamine discharge or locomotor function demonstrating the presence of nicotine receptors on dopaminergic segment might be concerned in attenuating the addictive property of nicotine [27]. With the help of microdialysis, a method after analysis shows the presence of miniature number of neurotransmitters in exact CNS areas. With the help of these findings scientist had revealed nicotine enhances the discharge of dopamine in the NAcs. The dopamine discharge in case of nicotine is quite similar to other addictive substances like heroin cocaine etc. [28].

\section{Other biological theories related to addiction to nicotine}

\subsection{Action of on cholinergic transmission}

Many other investigators had also provided even more ideas as about action of nicotine in brain. The production of nicotine central effect is through nAChRs. The cholinergic receptors are one of the largest receptors and contain numerous elements identified as subunits. Nicotinic receptors consist of XII subunits which participate in autonomic communication [29]. The nicotinic receptors located in brain are diverse gated-ion channels and mainly constitute of 5 subunits. Diverse association of receptors makes its different combinations, which differ in conditions of affinity and confined inside the brain. The $b$ subunit had been newly concerned for showing addictive property of nicotine [30]. The maximum sensitivity by nicotine is on alpha 4-beta 2 subunit arrangement. By means of extremely complicated bioengineering instrumentation, a new strain of mice was generated and the beta 2 subunit which was responsible for addictive property was removed. These mice were called "knockout" mice. Knockout mice were not able to create b subunit due to which mice was not showing nicotine self administration property [31].

These findings depicts that the beta subunit must have a crucial function in eliciting the pleasing property of nicotine. Results associated with these consequences provided researchers important innovative illustrations regarding nicotine action in the brain. These Illustrations eventually may show the way for improved treatment options for nicotine addiction [32].

Repetitive consumption of tobacco produces boli of nicotine transported in the CNS, through stable plasma concentration maintained during the smoking period. [33]. Thus initial intensity of nicotine shall maintain amount of $\mathrm{nAChR}$ in desensitized condition, whereas the enduring concentration of nAChR is presented for creation by nicotine boli, if suitable intensity is reached. This makes clear how consumers maneuver their blood nicotine intensity to attain stability inactivation against commencement. It too depicts why the primary cigarette of the day is the mainly rewarding, as during the night moderation allows a significant improvement from $\mathrm{nAChR}$ inactivation [34]. This additionally recommends in sleeping smoker, plasma intensity of nicotine reduces in plasma with recovery of the nicotinic receptors for their active useful condition. In the dawn there is increase in the intensity of nAChR active receptor sites, thus it may to growth of withdrawal indications and craving [35]. Furthermore, according to accepted research, amount of binding sites of nicotinic receptors are elevated in the brain of tobaco consumer observed after examination in the head of animals administered nicotine every day for one week. The other types of nAChR subtypes may too be sensitized but only at elevated applications of nicotine [36]. There are contradictory statements regarding the useful category of nAChR subsequent to chronic action, through increased, declined and unaffected intensity of sensitivity being depicted. Constant revelation to nicotine quickly causes inactivation of nAChR but it can also produce unending result. The alpha 4 beta2 nAChR is more desensitized than alpha3 beta2 nAChR [37]. Accurate reason and means of sensitization stay contentious. Moreover, changeable character of nAChR sensitization is at chances by means of an extended period vulnerability to waning signifying with additional, 
long period alteration in the CNS [38].

\subsection{Action on GABAergic transmission}

The interface among nicotine and GABAergic system was currently revealed. With this research numerous electrophysiological methods have confirmed that agonists of nicotinic rouse discharge of GABA from animal's brain parts plus it was $\mathrm{Ca} 2+$ dependent [39]. In recent times, the measures of tobacco on ventral tagmental GABAergic interneuron modulated the mesolimbic dopamine activity [40]. With the help extracellular recording methods in rat brain parts, nicotine enhanced the releasing speed of dopamine and non-dopamine neurons, and it is exciting to know that the firing of dopaminergic neuron was more vital. Findings related to this depicts that nicotine enhances the discharge tempo of dopaminergic neurons of VTA as well the GABA-ergic neurons [41]. It could be concluded that it might be an essential site for action of nicotine on CNS. The decreased vigorous reaction in the non-dopamine GABAergic neurons produces distinct inactivation. It ultimately may guide to desensitization of dopamine neurons thus assisting further controlled raise in result of mesolimbic dopamine neurons to nicotine [42].

Various researches encompass also revealed that nicotine also potentiates discharge of Nor- adrenalin in various segments of the brain, as well as nicotine shows its action mainly on locus ceruleus intensity [43]. It was too depicted that in hippocampus there is maximum desensitization of nicotine enthused NA as soon as forty minutes and continues for at least one and half hour and after that inactivation turn out to be a dominant method. This makes speculations that this might be mediating the 'reassuring' property of nicotine ingestion. Still, further more researches are needed to know the action of nicotine on different receptors and also to understand the aspects of its dependence [44].

\subsection{Action on Serotonergic transmission}

Tobacco ingestion and daily nicotine exposure lowers the application of 5-HT in hippocampus. It could be mainly because of selective enhancement of concentration of 5 HT1A receptors in brain segment [45]. Various researchers have concluded after their work that hippocampus obtains serotonergic innervations from the median raphe nucleus. Inhibition of 5- HT discharge within this fraction of hippocampus conveys anxiolytic reaction to nicotine as soon as injected locally by microinjection into the dorsal hippocampus [46]. Action of nicotine on 5-HT could not be easy to separate present on dopaminergic neurons. The property of nicotine abandonment on dopamine discharge in the CNS may improved through revelation to demanding stimulus and may trigger the function of anxiety as a feature in tobacco ingestion, in addition to the function of nicotine on decreasing the action by its action on 5-HT neurons inside hippocampus. At present, very les proof available in support of participation of 5- HT system in the affirmative reinforcing action of nicotine. There are a few proves available about the involvement serotonin in the negative craving action of nicotine abandonment [47]. In recent times, the demonstrations on animal models have depicted common relationship among nicotine withdrawal and opiate asceticism syndrome. The researches done so far suggested that nicotine stimulus provoke the discharge of endogenous opioid peptides in different brain segment ensuing in over creation of opiate receptors. The result obtained might be similar to opiate dependence. Rapid extinction of the order of nicotine enthused endogenous opioid discharge and opiate receptor inspiration force then impetuous an opiate asceticism -like state [48].

\subsection{Action on Monoamineoxidase enzymes lo- cated in brain}

A current research depicts that cotinine enhance nicotinic receptors to enhance the discharge of DA in a calcium-dependent way. Newer techniques such as advanced neuro- imaging technology had made it feasible to really observe spectacular action of tobacco ingestion on the brain of an conscious and behaving mammals [49]. Positron emission tomography (PET) has provided scientists reveal that tobaco ingestion effect a noticeable reduction in intensity of an essential enzyme, MAO (monoamineoxidase), which is accountable for infringement of dopamine. The reduction in two types of this enzyme MAO- A and B, consequences in elevated level of dopamine. Investigations had revealed that even though nicotine causes elevation in brain dopamine, nicotine does not modify MAO intensity [50]. These guides to opportunity of a new constituent of cigarette smoke additional to nicotine could be reducing the MAO. Thus, are many possibilities by which the smoking may alter the neurotransmitter level for satisfaction and reward [51].

In current years findings on hereditary constituent of cigarette ingestion have enhance our perceptive of nicotine reliance. CYP2A6 is enzyme accountable for maximum deactivation of nicotine in humans. CYP2A6 is conscientious for modulating tobacco-associated procarcinogens for example the nitrosamines. Inherited distinction in CYP2A6 gene might defend persons from fetching nicotine reliant smokers. New conclusion proposes that blocking this gene deficiency through blocking CYP2A6 reduces nicotine metabolism. More investigations are required in organize way to get better indulgent of how hereditary dissimilarity in CYP2A6 changes threat for nicotine reliance and decreases nicotine utilization [52].

\section{Conclusion}

Nicotine is a psychoactive substance easily available in all corners of the world. Nicotine as whole serve as a major enforcer in the form of tobacco, cigarettes etc. in the human beings. It shows a complex mechanism involving neuronal system in the brain. Various studies have shown that nicotine increases the activity of dopaminergic system, its release leading to the craving and withdrawal effect. Further the study of nicotine on different brain 
system such as cholinergic, serotonergic, GABAergic and noradrenergic may also be involved in evoking pleasurable effect of nicotine. Molecular mechanism of nicotine addiction is still a challenging task for the researchers as so many brain systems are involved in it. The combination of molecular modern techniques with behavioral analysis may provide a exact mechanism and knowledge about nicotine addiction.

\section{Acknowledgment}

The authors are thankful to Columbia institute of Pharmacy for providing necessary facility for completion of work.

\section{Conflict of Interest}

The author declares no conflict of interest.

\section{References}

1. Tiwari RK, Shukla SS, Roy A, Satapathy T, Pandey R. Role of Serotonin in Relapse to Nicotine addiction: An Overview. Advance Research in Pharmaceuticals \& Biologicals. 2012;2(II):157-66.

2. Goshman LM. Clinical Toxicology of Commercial Products. Journal of Pharmaceutical Sciences. 1985;74:1139-55.

3. Cunha OT, Rego AC, Oliveira CR. Cellular and molecular mechanisms involved in the neurotoxicity of opioid and psychostimulant drugs. Brain Res Rev. 2008;58(1):192-208.

4. Zhu PJ, Chiappinelli VA. Nicotine modulates evoked GABAergic transmission in the brain. J Neurophysiol. 1999;82:3041-5.

5. Perkins K, Stitzer M, Lerman C. Medication screening for smoking cessation: a proposal for new methodologies. Psychopharmacology. 2006;184(3-4):628-36.

6. Hurst R, Rollema H, Bertrand D. Nicotinic acetylcholine receptors: from basic science to therapeutics. Pharmacol Ther. 2013;137:22-54.

7. Di Chiara G. Role of dopamine in the behavioural actions of nicotine related to addiction. European journal of pharmacology. 2000;393(1):295-314-344.

8. Bardo MT, Green TA, Crooks PA, Dwoskin LP. Nornicotine is self-administered intravenously by rats. Psychopharmacology. 1999;146(3):290-6.

9. Kollins SH, McClernon FJ, Fuemmeler BF. Association between smoking and attention-deficit/hyperactivity disorder symptoms in a population-based sample of young adults. Arch Gen Psychiatry. 2005;62:1142-7.

10. Heishman SJ, Kleykamp BA, Singleton EG. Meta-analysis of the acute effects of nicotine and smoking on human performance. Psychopharmacology. 2010;210:453-69.

11. Jain R, Varma S, Mohan D. Effects of selective dopaminergic drugs in nicotine tolerant rats. Drug Alcohol Depend. 2001;63:72.

12. Hopkins TJ, Rupprecht LE, Hayes MR, Blendy JA,
Schmidt HD. Galantamine, an acetylcholinesterase inhibitor and positive allosteric modulator of nicotinic acetylcholine receptors, attenuates nicotine taking and seeking in rats. Neuropsychopharmacology. 2012;37:2310-21.

13. Kushner HI. Toward a cultural biology of addiction. BioSocieties. 2010;5(1):8-24.

14. Addicott MA, et al. Smoking withdrawal is associated with increases in brain activation during decision making and reward anticipation: a preliminary study. Psychopharmacology (Berl). 2012;219(2):563-73

15. Gotti C, Clementi F, Fornari A, Gaimarri A, Guiducci S, Manfredi I, et al. Structural and functional diversity of native brain neuronal nicotinic receptors. Biochem Pharmacol. 2009;78:703-11.

16. 16. Satoshi I. Brain reward circuitry beyond the mesolimbic dopamine system: A neurobiological theory. Neurosci Biobehav Rev. 2010;35(2): 129-50.

17. Christopher C Lapish, Sven K, Daniel D, Antonieta L, Jeremy K Seamans. The ability of the mesocortical dopamine system to operate in distinct temporal modes. Psychopharmacology. 2007;191(3): 609-25.

18. Centers for Disease Control and Prevention (US); National Center for Chronic Disease Prevention and Health Promotion (US); Office on Smoking and Health (US). How Tobacco Smoke Causes Disease: The Biology and Behavioral Basis for Smoking-Attributable Disease: A Report of the Surgeon General. Atlanta (GA): Centers for Disease Control and Prevention (US); 2010. 4, Nicotine Addiction: Past and Present.

19. Christian L, Mark AU. The Mechanistic Classification of Addictive Drugs. PLoS Med. 2006;3(11):e437.

20. Bryon AD. Neurobiological Processes in Drug Reward and Addiction. Harv Rev Psychiatry. 2004;12(6):305-20.

21. Alfred JR, Eric JN. Transcriptional and Epigenetic Mechanisms of Addiction. Nat Rev Neurosci. 2011;12(11): 623-37.

22. Hugo JO, David CG, Ernestina HG, Gerardo BM. The Role of Dopamine and Its Dysfunction as a Consequence of Oxidative Stress. Oxid Med Cell Longev. 2016;2016: 9730467.

23. Buchta WC, Riegel AC. Chronic cocaine disrupts mesocortical learning mechanisms. Brain Research. 2015;631-5.

24. Deutch AY, Roth RH. The determinants of stress-induced activation of the prefrontal cortical dopamine system. Progress in Brain Research. 1990;85:367-403.

25. Schmitt KC, Rothman RB, Reith ME. Nonclassical pharmacology of the dopamine transporter: atypical inhibitors, allosteric modulators, and partial substrates. Journal of Pharmacology and Experimental Therapeutics. 2013;346(1):2-10.

26. Calabresi P, Picconi B, Tozzi A, Di Filippo M. Dopamine-mediated regulation of corticostriatal synaptic plasticity. Trends in Neurosciences. 2007;30(5):211-9

27. Salas R, Cook KD, Bassetto L, De Biasi M. The alpha3 and beta4 nicotinic acetylcholine receptor subunits are necessary for nicotine-induced seizures and hypolocomotion in mice. Neuropharmacology. 2004; 47(3):401-7.

28. Nick C, Ortiz BA, Heidi C, O’Neill, Michael J, Marks, et al. Varenicline Blocks $\beta 2 *$-nAChR-Mediated Response 
and Activates $\beta 4^{*}$-nAChR-Mediated Responses in Mice In Vivo Nicotine. Tob Res. 2012;14(6): 711-9.

29. Bernard LF, Steven RG. Effects of nicotine in experimental animals and humans: an update on addictive properties. Handb Exp Pharmacol. 2009; (192): 335-67.

30. Neal LB. Pharmacology of Nicotine: Addiction, Smoking-Induced Disease, and Therapeutics. Annu Rev Pharmacol Toxicol. 2009; 49:57-71.

31. Charles RW, Michael JM, Sharon RG. Effectiveness of Nicotinic Agonists as Desensitizers at Presynaptic $\alpha 4 \beta 2$ - and $\alpha 4 \alpha 5 \beta 2-$ Nicotinic Acetylcholine Receptors. Nicotine Tob Res. 2014;16(3): 297-305.

32. Arthur L.B, Alexey G.M, Michael SM, Trinh L, Meaghan N, Lidia L, Jennifer S, Catherine AS, Jed ER, Mark AM. Brain Nicotinic Acetylcholine Receptor Availability and Response to Smoking Cessation Treatment A Randomized Trial. JAMA Psychiatry. 2014;71(7):797-805.

33. Rachel EP, Robin AJL. Cellular Events in Nicotine Addiction Semin Cell Dev Biol. 2009;20(4): 418-31.

34. Wang H, Sun X. Desensitized nicotinic receptors in brain. Brain Res Brain Res Rev. 2005;48(3):420-37.

35. Yates SL, Bencherif M, Fluhler EN, Lippiello PM. Up-regulation of nicotinic acetylcholine receptors following chronic exposure of rats to mainstream cigarette smoke or alpha 4 beta 2 receptors to nicotine. Biochem Pharmacol. 1995;50(12):2001-8.

36. Laura M, Emanuele S, Enrico C. Regulation of GABA release by nicotinic acetylcholine receptors in the neonatal rat hippocampus. J Physiol. 2001;536(Pt 1): 89-100.

37. Meaghan C, Creed NRN, Kelly RT. VTA GABA neurons modulate specific learning behaviors through the control of dopamine and cholinergic systems. Front Behav Neurosci. 2014;8:8.

38. Danyan M, Keith G, Daniel S, Mc Gehee. Nicotine potentiation of excitatory inputs to VTA dopamine neurons J Neurosci. 2011; 31(18): 6710-20.

39. Pidoplichko VI, DeBiasi M, Williams JT, Dani JA. Nicotine activates and desensitizes midbrain dopamine neurons. Nature. 1997;390(6658):401-4.

40. Jain R, Mukherjee K. Biological basis of nicotine addiction. Indian Journal of Pharmacology. 2003;35:281-9.

41. Jin HP, Soo K, Areum D, Ji HK, Ah JK. Symptomatic bradycardia due to nicotine intoxication. Rev Bras Ter Intensiva. 2018; 30(1):121-6.

42. Jiloha RC. Biological basis of tobacco addiction: Implications for smoking-cessation treatment. Indian J Psychiatry. 2010;52(4):301-7.

43. Galindo CL, Hernandez LS, Galarraga E, Tapia D, Bargas J, Garduno J, et al. Serotoninergic dorsal raphe neurons possess functional postsynaptic nicotinic acetylcholine receptors. Synapse. 2008; 62(8):601-15.

44. Munir GK, Thomas JG. Nicotine Modulation of Fear Memories and Anxiety: Implications for Learning and Anxiety Disorders. Biochem Pharmacol. 2015; 97(4): 498-511.

45. Yue X, Edward FD. Tobacco/Nicotine and Endogenous Brain Opioids Prog Neuropsychopharmacol. Biol Psychiatry. 2008;32(5): 1131-8.

46. George EB, Alexander I, Valentina EM. Beneficial effects of nicotine, cotinine and its metabolites as potential agents for Parkinson's disease. Front Aging Neuro- sci. 2014; 6: 340.

47. Joanna SF, Nora DV, Gene JW, Naomi P, Jean L, Colleen $\mathrm{S}$, et al. Brain monoamine oxidase A inhibition in cigarette smokers. Proc Natl Acad Sci U S A. 1996;93(24): 14065-9.

48. Neal LB. Pharmacology of Nicotine: Addiction, Smoking-Induced Disease, and Therapeutics. Annu Rev Pharmacol Toxicol. 2009;49:57-71.

49. Tyndale RF, Pianezza ML, Sellers EM. A common genetic defect in nicotine metabolism decreases risk for dependence and lowers cigarette consumption. Nicotine Tob Res. 1999; 1(Suppl 2):S63-7.

50. Julie AT, Jeffrey AH, Dedra B, Barbara VH, Patricia NH, Rachel FT. Variation in CYP2A6 and nicotine metabolism among two American Indian tribal groups differing in smoking patterns and risk for tobacco-related cancer. Pharmacogenet Genomics. 2017; 27(5):169-78.

51. Bierut LJ, Stitzel JA, Wang JC, Hinrichs AL, Grucza RA, Xuei X, et al. 2008. Variants in nicotinic receptors and risk for nicotine dependence. Am J Psychiatry. 165:1163-71.

52. Johnstone EC, Yudkin PL, Hey K, et al. Genetic variation in dopaminergic pathways and short-term effectiveness of the nicotine patch. Pharmacogenetics. 2004;14(2):83-90. 